\title{
Evaluation of a Modified Scleral Contact Lens as a Riboflavin Delivery Device for Corneal Collagen Crosslinking
}

\author{
Ricardo Torres Soares*, Neil F. Novo, Willy Marcus França \\ Faculty of Medical and Health Science, Catholic University of São Paulo, São Paulo, Brazil \\ Email: *drricard@icloud.com
}

How to cite this paper: Soares, R.T., Novo, N.F. and França, W.M. (2017) Evaluation of a Modified Scleral Contact Lens as a Riboflavin Delivery Device for Corneal Collagen Crosslinking. Open Journal of Ophthalmology, 7, 273-280.

https://doi.org/10.4236/ojoph.2017.74036

Received: September 2, 2017

Accepted: October 28, 2017

Published: October 31, 2017

Copyright (c) 2017 by authors and Scientific Research Publishing Inc. This work is licensed under the Creative Commons Attribution International License (CC BY 4.0).

http://creativecommons.org/licenses/by/4.0/

\begin{abstract}
Introduction: Keratoconus is a complex corneal disease that reduces visual acuity by progressively modifying the corneal shape and thickness, usually producing myopia and irregular astigmatism. Corneal collagen crosslinking with riboflavin + ultraviolet-A radiation (CXL) has become a widely accepted treatment for progressive keratoconus. During CXL, riboflavin administration is performed by repeated manual instillation of solution drops on the cornea for 30 minutes, a procedure that is often uncomfortable for many patients and that consumes surgical facilities and staff resources. In this study, especially modified scleral contact lenses (MSCL) were employed for delivering riboflavin to the cornea during CXL. Objective: The study aimed at evaluating the safety and efficacy of MSCL as a drug delivery system, verifying if anterior chamber flare confirms riboflavin penetration and describes the impact on patient comfort and optimization of surgical staff and facility resources. Material and Method: This study included 8 eyes of 6 patients aged $16-25$ years old with history of progressive keratoconus. After mechanical removal of corneal epithelium, the concave surface of the modified scleral contact lens was filled with riboflavin solution and the lens was placed on the patient's eye during 30 minutes. The lens design allows the formation of a riboflavin layer between the lens and the exposed corneal stroma to facilitate riboflavin penetration. Patients with lens were allowed to stand up and wait for the second UVA phase outside the surgical room. Riboflavin diffusion was confirmed by biomicroscopic examination of the corneal stroma and anterior chamber with the lens in place. Patients returned and the lens was removed before UV-A irradiation at $3 \mathrm{~mW} / \mathrm{cm}^{2}$ for 30 minutes. Statistical analysis was performed by comparing the following parameters of each patient pre- and post-CXL: Spherical equivalent (Sph.Eq.), Mean simulated keratometry (SimK-m) and
\end{abstract}


corrected distance visual acuity (CDVA) using the Wilcoxon method for non-parametric data $(p<0.05)$. Results: The MSCL allowed patients to be transferred from the surgical room to wait for corneal impregnation with riboflavin. The MSCL was effective in delivering riboflavin to the cornea as confirmed by biomicroscopic examination of the cornea and anterior chamber. No intraoperative or postoperative complications were observed. MSCL use improved the patient comfort and reduced the burden on surgical staff and facilities. All analyzed parameters showed statistically significant differences pre- and post-CXL: Sph.Eq. $p=0.018$ (Median: -2.50 ; Average: -2.52 ); SimK-m $p=0.006$ (Median: 47.92; Average: 45.56). CDVA $p=0.012$ (Median: -0.45 ; Average: 0.42 ). Conclusion: The MSCL is a safe and efficacious device for riboflavin delivery during CXL. The present study permits slit lamp observation of anterior chamber flare to confirm riboflavin penetration, and provides added safety and comfort for the patient and convenience to healthcare providers by optimizing the use surgical facilities and staff. Keratometric, visual and refractive results were similar to those reported in the literature for CXL with manual riboflavin instillation. Additional studies with larger numbers of patients are needed to confirm the study findings.

\section{Keywords}

Keratoconus, Riboflavin, UVA, Rigid Contact Lens, Cross-Linking

\section{Introduction}

Corneal integrity is an essential component for good quantitative and qualitative vision. Keratoconus is a complex and progressive corneal disease whose origin has not been fully clarified. It decreases visual acuity by producing progressive changes to the shape, thickness and transparency of the cornea, resulting in altered visual perception [1]. Corneal transplants are still the most common therapeutic option for advanced cases such as keratoconus stages III and IV [2].

In recent years, new keratoconus treatment modalities have arisen, such as implantation of intrastromal corneal ring segments (ICRS) and corneal collagen cross-linking (CXL). ICRS implantation in keratoconus aims at reducing corneal curvature and irregularities, thus improving visual acuity [3] [4]. CXL with riboflavin and ultraviolet A (UV-A) irradiation would initiate a significantly more apoptosis of the anterior stroma cells when comparing with corneal scrape (Epi-off) with RBV isolated or UV-A isolated in experimental animals (rabbits) [5]. Comparative study between Epi-off versus Epi-on, both associated to RBV + UV-A, demonstrated 70\% superior efficacy of Epi-off in the regression or stabilization of Keratoconus in children (12 -18 years old) when compared to Epi-on [6] [7].

Currently, CXL with riboflavin associated to UV-A is a safe and effective option of surgical treatment for keratoconus and post-refractive surgery corneal 
ectasia, demonstrating mechanical and biochemical stability of the stromal tissue in the majority of cases [8]. CXL may be performed with epithelium removal (Epi-off) or without (Epi-on). On the Epi-off technique, epithelial removal is carried out prior to corneal soakage with a dextran-based $0.1 \%$ riboflavin solution, followed by irradiation with UV-A light for $30 \mathrm{~min}$ with an intensity of 3 $\mathrm{mW} / \mathrm{cm}^{2}$. Adequate impregnation of the cornea with the riboflavin solution is essential for the safety and efficacy of the procedure and requires manual administration of riboflavin solution drops every 5 minutes during 30 minutes or until stromal saturation can be confirmed through slit lamp examination [8]. Riboflavin is also applied every 5 minutes throughout the UV-A irradiation phase. For a period of 1 hour or longer, the patient must remain static on the surgical table in dorsal decubitus position and wear a blepharostat. As a result, the cornea soaking stage of the CXL procedure is often uncomfortable for the patient and a tedious time for the surgeon. Additionally, the CXL procedure entails increased costs as the surgical room remains occupied and surgeon and staff are unable to perform other duties throughout the entire procedure time [9].

The lens designed allows the formation of a riboflavin reservoir between the lens and the cornea to facilitate penetration and eliminate the need for repeated manual administration of riboflavin. The present study aimed to evaluate the safety and efficacy of MSCL as a drug delivery system and impact of using a modified scleral contact lens (MSCL) (Figure 1) during CXL procedure and

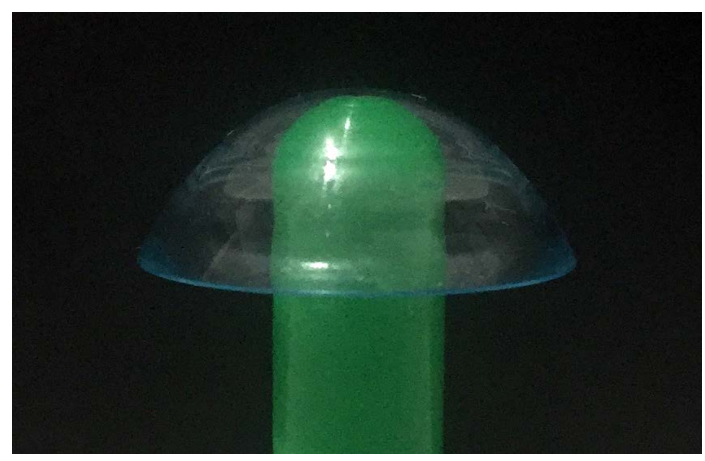

(a)

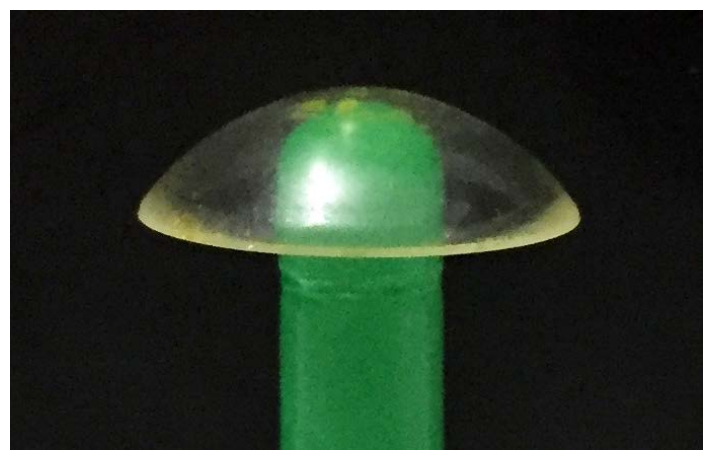

(b)

Figure 1. (a) Shows the modified scleral contact lens; (b) Shows the conventional scleral lens. 
check if anterior chamber flare confirms riboflavin penetration into the cornea.

\section{Material and Method}

In the present study, we enrolled 8 eyes of 6 patients ( 5 males, 1 female), aged 15 to 21 years (mean 17 years old), with history of progressive keratoconus documented by sequential corneal topography, minimum corneal thickness of 420 $\mu \mathrm{m}$ and indication for CXL treatment (Table 1). Ethical principles for medical research involving human subjects were observed and in accordance with the Declaration of Helsinki of the World Medical Organization.

After antisepsis, placement of sterile drapes and blepharostat, the corneas were submitted to mechanic epithelial removal in an area of approximately 9 $\mathrm{mm}$ in diameter [6] [7] (Figure 2) under topical anesthesia. A sterile, disposable modified scleral contact lens (MSCL) (Mediphacos S/A, Brazil) was filled with dextran-based $0.1 \%$ riboflavin sterile solution and placed on the patient's eye for 30 minutes (Figure 3(a) and graph in Figure 3(b)).

The MSCL is a single-use drug delivery device made from polymethylmethacrylate (PMMA), having an overall diameter of $16.5 \mathrm{~mm}$ and a plano optical dioptric power. The MSCL is designed so that its edge rests on the conjunctiva overlying the sclera and its optical zone vaults the entire cornea and limbus, thus

Table 1. Preoperative parameters measurements of patients' Sph-E, SimK-m and CDVA.

\begin{tabular}{cccc}
\hline Eyes & Sph-E & SimK-m & CDVA \\
\hline 1 & -4.13 & 51.09 & 0.2 \\
$2 \mathrm{a}$ & -0.38 & 42.10 & 0.8 \\
$\mathrm{2b}$ & 0.00 & 45.05 & 0.5 \\
$\mathrm{3a}$ & -6.50 & 57.10 & 0.1 \\
$3 \mathrm{~b}$ & -1.13 & 47.55 & 0.5 \\
4 & -2.50 & 48.05 & 0.4 \\
5 & -2.50 & 47.80 & 0.66 \\
6 & -3.00 & 57.75 & 0.2 \\
\hline
\end{tabular}

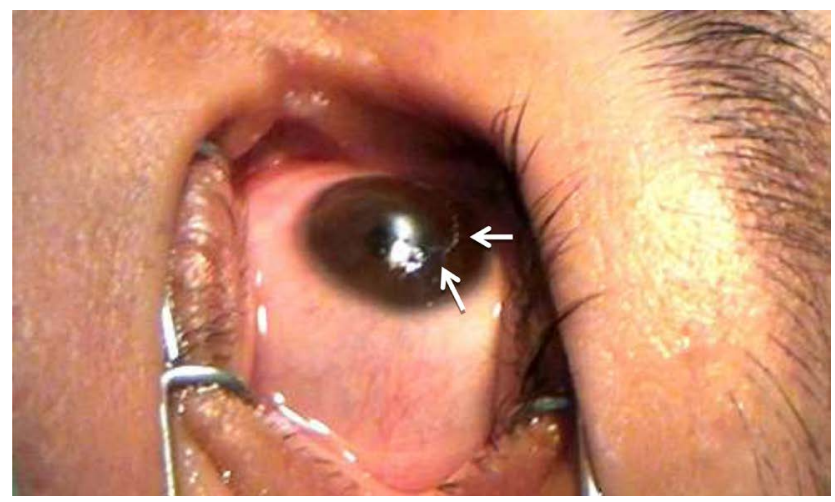

Figure 2. The de-epithelialized cornea (Epi-off) (arrows) prepared to receive the lens with RBV. 


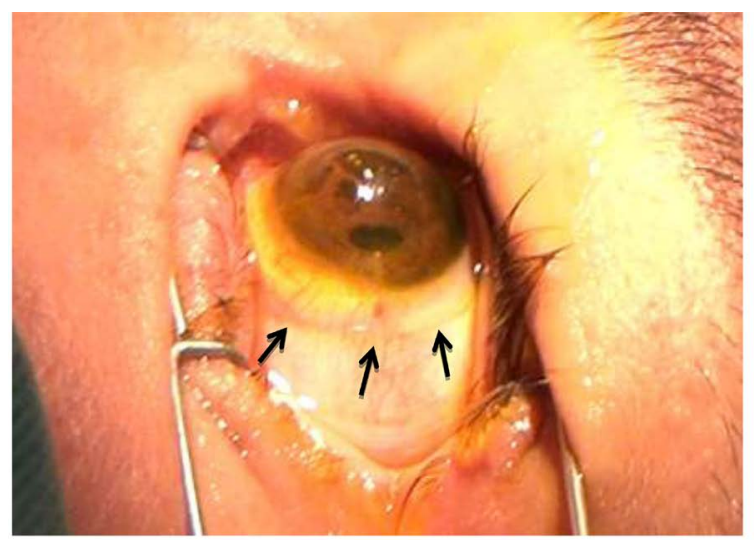

(a)

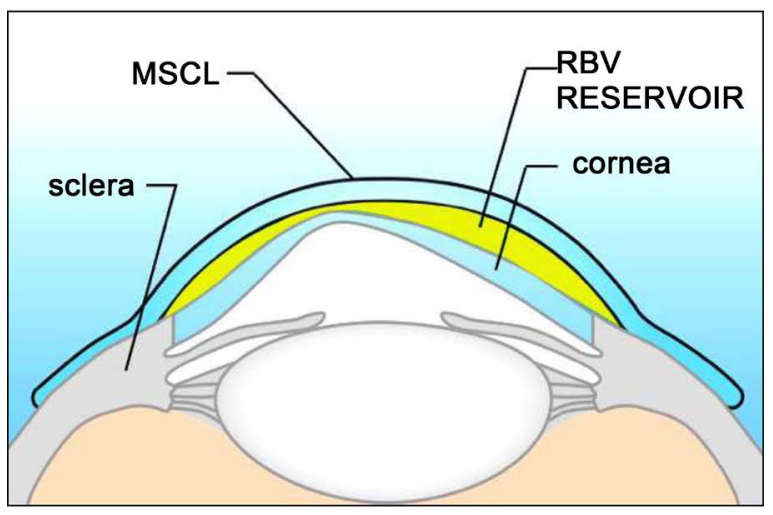

(b)

Figure 3. (a) Shows the MSCL placed on the eye and formation of the riboflavin reservoir; (b) Shows the MSCL placed on the eye and formation of the riboflavin reservoir.

creating a sealed liquid reservoir that when filled with riboflavin allows constant contact between the solution and the corneal tissue. The MSCL is available in one size only and is designed to cope with individual variations in scleral or corneal curvature, elevation or shape.

Following placement of the riboflavin-filled MSCL, the blepharostat was removed and the patients transferred from the surgical room to an adjoining room where they remained seated and were examined with a slit lamp every $15 \mathrm{mi}$ nutes to observe corneal impregnation. Patients then returned to the surgical room where following another antisepsis, blepharostat placement and removed de MSCL; the corneas were irradiated with ultraviolet-A using UVX-1000 light source (IROC, Switzerland) for 30 minutes with an intensity of $3 \mathrm{~mW} / \mathrm{cm}^{2}$.

Postoperatively, patients received a bandage soft contact lens for 5 days and were prescribed gatifloxacin $(3 \mathrm{mg} / \mathrm{ml}) /$ prednisolone acetate $(10 \mathrm{mg} / \mathrm{ml})(\mathrm{Zy}-$ pred - Allergan) 3 times a day for 7 days, then 2 times a day for additional 7 days; lubricating eye drops (Refresh, Allergan) 3 times a day for 30 days; and paracetamol tablets in case of ocular pain.

Statistical analysis was performed comparing the following parameters of each patient pre and post CXL: Spherical equivalent, SimK-mean, corrected distance visual acuity using the Wilcoxon method for non-parametric data [9]. The sta- 
tistical analysis confidence interval was $5 \%(p<0.05)$.

\section{Results}

No intraoperative or postoperative complications were observed. While wearing the MSCL, patients could be transferred from the surgical room to an adjoining examination room where they remained seated without a blepharostat for 30 minutes. Patients did not complain of pain or discomfort and no MSCL was spontaneously displaced or dislodged during this time. The MSCL was effective in delivering riboflavin to the cornea. Solution diffusion in the stroma and anterior chamber flare were visible on biomicroscopy after 30 minutes with the lens in place. While patients waited for riboflavin penetration they did not require close supervision from surgical personnel, so surgical room, surgeon and staff were free to perform other duties.

Final postoperative visits were done 11.6 months after surgery on average (range 3 to 15 months). Preoperative and Postoperative Sph.Eq., SimK-m and CDVA all showed statistically significant differences (Table 2).

Sph. Eq: $p=0.018$ (Median: -2.50 ; Average: -2.52 ).

SimK-m: $p=0.006$ (Median: 47.92; Average: 45.56).

CDVA: $p=0.012$ (Median: 0.45; Average: 0.42 ).

\section{Discussions}

CXL is an established method that has quickly gained acceptance for the treatment of progressive keratoconus. It has been demonstrated that the association of Epi-off, riboflavin + UVA is a safe and effective surgical procedure to halt keratocous progression in most cases [10] [11] [12]. Arora et al., 2012, obtained a significant CDVA improvement in patients affected with Keratoconus using CXL with Epi-off $(p=0.003)$, as well as a significant improvement in SimK-m ( $p$ $=0.004)$, when compared to two treatment moments, pre- and post-CLX [10]. Lesniak et al., 2014, have also obtained statistically significant results $(p=0.03)$ in CDVA of patients with Keratoconus using CXL + Epi-on with a six-month

Table 2. The measurements of the study parameters S-Sph, SimK-m and VA after lens intervention.

\begin{tabular}{cccc}
\hline Eyes & S-Sph & SimK-m & VA \\
\hline 1 & -2.75 & 51.05 & 0.33 \\
$2 \mathrm{a}$ & 0.00 & 41.25 & 1.00 \\
$2 \mathrm{~b}$ & 2.00 & 44.45 & 0.66 \\
$3 \mathrm{a}$ & -0.75 & 51.35 & 0.40 \\
$3 \mathrm{~b}$ & -0.50 & 43.90 & 0.66 \\
4 & 1.50 & 46.40 & 0.66 \\
5 & -0.88 & 45.40 & 0.66 \\
6 & -3.00 & 53.35 & 0.40 \\
\hline
\end{tabular}


follow-up of their patients. They have also presented a significant improvement in Simk-m with a flattening of 0.9 diopter $(p=0.01)$ [11]. Experimental studies in porcine eyes showed that riboflavin penetration in the corneal stroma is greater with the use of protocols and assisted iontophoresis, probably due to a greater riboflavin diffusion into the corneal stroma [12]. These authors conducted a comparative study of Keratoconus treated with CXL with Epi-off and Epi-on. The study showed a significant difference in VA only in the Epi-off group with a follow-up period of only 12 and 24 months $(p<0.05)$. On the other hand, the second group Epi-on presented significant improvement within 6 months and also 12 and 24 months $(p<0.05)$. Regarding SimK-m, there was a statistically significant difference in both groups within 12 and 24 months $(p<$ 0.05 ) [12]. Others authors attest that Epi-on presented only 70\% Epi-off efficacy regarding the decrease of Keratoconus progression and stabilization [6] [7].

The present study, in line with most of the literature, employed CXL with Epi-off and presented statistically significant differences in the results of Sph.Eq. $(p=0.018)$, SimK-m $(p=0.006)$ and CDVA $(p=0.012)$ using the MSCL drug delivery device manufactured for this study. This device allowed possibility of cornea saturation within 30 minutes of wear, permitting the patient examination with a slit lamp to visualize riboflavin in the anterior chamber. Given that in this study all the CXL indication criteria were rigorously observed, as described by Seiler T et al., 2017 (Epi-off, riboflavin for 30 minutes, UVA for 30 min with 370 $\mathrm{nm}$ wave length and cornea thickness above 400 microns), the use of the MSCL was shown to be safe and efficient method of delivering riboflavin to the cornea, producing statistically significant refractive, topographic and visual changes comparable to standard CXL with manual riboflavin administration [8].

The MSCL material and design allows its production in large scale and at reduced cost compared to standard scleral contact lenses, which makes it suitable for the single-use regimen. To effectively avoid infection, it is essential that the MSCL is presented to be sterile since it is applied intra-operatively over a de-epithelized cornea that is prone to contamination. The MSCL acts as a protective corneal shield and avoids any external contaminant to come in contact with the cornea, thus potentially improving the safety profile of the CXL procedure.

The ability to move the patient to another room while wearing the MSCL and without blepharostat allows the patient to wait more comfortably in a seated position and engage in activities such as reading, texting or just resting. This improves the patient's experience during CXL and frees surgical staff and room for other activities, thus contributing to a reduction of the procedure cost by optimization of surgical resources.

In this study, MSCL can be easily used in a patient for the first 30 minutes of treatment with no statistical difference from literature. This would suggest a reduction in the corneal impregnation time with the use of the MSCL due to the permanent contact between the solution and the cornea. But the lens design al- 
lows the formation of a riboflavin reservoir between the lens and the cornea that facilitates penetration and eliminates the need for repeated manual administration of riboflavin. It also confirms that anterior chamber flare presented riboflavin penetration and suggested a good impact on patient comfort. Given the small patient sample, this study continues with larger number of patients.

\section{References}

[1] Touboul, D., Robinet-Perrin, A., Fournié, P. and Malecaze, F. (2016) Efficacy of Corneal Cross-Linking for the Treatment of Keratoconus. Journal Francais D'Ophtalmologie, 39, 308-314.

[2] Fuest, M., Yam, G.H., Peh, G.S. and Mehta, J.S. (2016) Advances in Corneal Cell Therapy. Regenerative Medicine, 11, 601-615.

[3] Chhadva, P., Yesilirmak, N., Cabol, F. and Yoo, S.H. (2015) Intrastromal Corneal Ring Segment Explanation in Patients with Keratoconus: Cause, Technique, and Outcomes. Journal of Refractive Surgery, No. 6, 392-397.

[4] Piñero, D.P. and Alio, J.L. (2010) Intracorneal Ring Segments in Ectatic Corneal Disease-A Review. Clinical \& Experimental Ophthalmology, 38, 154-167.

https://doi.org/10.1111/j.1442-9071.2010.02197.x

[5] Salomão, M.Q., Chaurasia, S.S., Sinha-Roy, A., Ambrósio Jr., R., Esposito, A., Sepulveda, R., Agrawal, V. and Wilson, S.E. (2011) Corneal Wound Healing after Ultraviolet-A/Riboflavin Collagen Cross-Linking: A Rabbit Study. Journal of Refractive Surgery, 27, 401-407.

[6] Raiskup, F., Veliká, V., Veselá, M. and Spörl, E. (2015) Cross-Linking in Keratoconus: "Epi-off" or "Epi-on"? Klinische Monatsblatter Fur Augenheilkunde, 232, 1392-1396.

[7] Eraslan, M., Toker, E., Cerman, E. and Ozarslan, D. (2016) Efficacy of Epithelium-Off and Epithelium-On Corneal Collagen Cross-Linking in Pediatric Keratoconus. Eye \& Contact Lens. (Epub Ahead of Print)

[8] Spoerl, E., Mrochen, M., Sliney, D., Trokel, S. and Seiler, T. (2007) Safety of UVA-Riboflavin Cross-Linking of the Cornea. Cornea, 26, 385-389. https://doi.org/10.1097/ICO.0b013e3180334f78

[9] Zonta, P.G., Moschou, K.A., Diakonis, V.F., Kymionis, G.D., Almaliotis, D.D., Karamitsos, A.P. and Karampatakis, V.E. (2012) Corneal Collagen Cross-Linking for Progressive Keratoconus in Pediatric Patients: A Feasibility Study. Journal of Refractive Surgery, 28, 793-799. https://doi.org/10.3928/1081597X-20121011-08

[10] Arora, R., Gupta, D., Goyal, J.L. and Jain, P. (2012) Results of Corneal Collagen Cross-Linking in Pediatric Patients. Journal of Refractive Surgery, 28, 759-762.

[11] Lesniak, S.P. and Hersh, P.S. (2014) Transepithelial Corneal Collagen Crosslinking for Keratoconus: Six-Month Results. The Journal of Cataract \& Refractive Surgery, 40, 1971-1979.

[12] Guzel, B. and Mukharram, B. (2016) Standard Corneal Collagen Crosslinking versus Transepithelial Iontophoresis-Assisted Corneal Crosslinking, 24 Months Follow-Up: Randomized Control trial. Acta Ophthalmologica, 94, e600-e606. 